\title{
On Measurement System for Frequency of Uterine Peristalsis
}

\author{
Ryosuke NISHIHARA $^{\dagger \mathrm{a})}$, Hidehiko MATSUBAYASHI ${ }^{\dagger \dagger}$, Tomomoto ISHIKAWA $^{\dagger \dagger \mathrm{c})}$, Nonmembers, \\ Kentaro MORI ${ }^{\dagger \dagger \mathrm{d})}$, and Yutaka HATA ${ }^{\dagger \mathrm{e})}$, Members
}

\begin{abstract}
SUMMARY The frequency of uterine peristalsis is closely related to the success rate of pregnancy. An ultrasonic imaging is almost always employed for the measure of the frequency. The physician subjectively evaluates the frequency from the ultrasound image by the naked eyes. This paper aims to measure the frequency of uterine peristalsis from the ultrasound image. The ultrasound image consists of relative amounts in the brightness, and the contour of the uterine is not clear. It was not possible to measure the frequency by using the inter-frame difference and optical flow, which are the representative methods of motion detection, since uterine peristaltic movement is too small to apply them. This paper proposes a measurement method of the frequency of the uterine peristalsis from the ultrasound image in the implantation phase. First, traces of uterine peristalsis are semi-automatically done from the images with location-axis and time-axis. Second, frequency analysis of the uterine peristalsis is done by Fourier transform for 3 minutes. As a result, the frequency of uterine peristalsis was known as the frequency with the dominant frequency ingredient with maximum value among the frequency spectrums. Thereby, we evaluate the number of the frequency of uterine peristalsis quantitatively from the ultrasound image. Finally, the success rate of pregnancy is calculated from the frequency based on Fuzzy logic. This enabled us to evaluate the success rate of pregnancy by measuring the uterine peristalsis from the ultrasound image.

key words: ultrasound images, female infertility, frequency of uterine peristalsis, Fourier transform, Fuzzy logic
\end{abstract}

\section{Introduction}

The number of infertility treatments is increasing year by year due to late marriage and late birth [1]. The demand for infertility treatment is also increasing. Infertility treatment is broadly divided into general infertility treatment and assisted reproductive technology (ART). A general infertility treatment is firstly performed. ART is considered as the next candidate when the general infertility treatment does not result in successful pregnancy, or the fertility symptoms are severe. ART is costly for the patients because it is not covered by the public insurance in Japan. Further, ART does not

Manuscript received August 25, 2020.

Manuscript revised February 17, 2021.

Manuscript publicized May 12, 2021.

${ }^{\dagger}$ The authors are with University of Hyogo, Kobe-shi, 6500047 Japan.

${ }^{\dagger \dagger}$ The authors are with Reproduction Clinic Osaka, Osaka-shi, 530-0011 Japan.

${ }^{\dagger+\dagger}$ The author is with National Institute of Technology Maizuru College, Maizuru-shi, 625-8511 Japan.

a)E-mail: sa20q012@sim.u-hyogo.ac.jp

b) E-mail: matsubayashihide@me.com

c) E-mail: tishikawa@ reposaka.jp

d)E-mail: k.mori@maizuru-ct.ac.jp

e) E-mail: hata@ sim.u-hyogo.ac.jp

DOI: 10.1587/transinf.2020LOP0003 guarantee pregnancy by one treatment, and it is sometimes necessary to repeat itself. As a result, the physical and financial burden on infertility patients becomes large. Some patients cannot choose ART by the economic reasons. Therefore, it is strongly required to improve the success rate of infertility treatment. The Office on Women's Health Trusted Source reported that about one-third of infertility cases can be attributed to female infertility while men's problems account for another third of infertility cases [2].

In female infertility cases, Embryo implantation is the primary factor with the limitation on ART. The uterine has movements called uterine peristalsis. The direction and the frequency of uterine peristalsis changes according to the phase of the menstrual cycle [3], [4]. The upward movement (from corpus to fundus) occurs in the ovulation phase to assist in sperm transport, and the downward movement (from fundus to corpus) occurs in the menstrual phase to assist in drain menstrual blood. In contrast, there are no movements in the implantation phase to wait for the fertilized egg. However, in fact, uterine peristalsis may actually occur in the implantation phase. The ultrasound examination [4]-[7] of the uterus has permitted the non-invasive identification and characterization of uterine contractile activity under physiological condition. Currently, the frequency of uterine peristalsis is evaluated by the number of times per minute on the ultrasound imagery. It is known a strong relationship between the frequency of uterine peristalsis in the implantation phase and the pregnancy rate. Moreover, it has been reported that the high frequency of uterine peristalsis implies the lower pregnancy rate [8]-[11]. In most cases, the frequency of uterus peristalsis is evaluated by visual analysis by the naked eye of the physician based on his/her experience. There is no clear criterion for measuring the frequency of uterus peristalsis. The frequency of uterus peristalsis in the embryo implantation greatly affects the pregnancy rate, thus the subjective evaluation is undesirable. Table 1 shows the relationship between the frequency of uterine peristalsis per minute in the implantation phase and pregnancy rate. This table shows that when the frequency of uterine peristalsis is more than 3.0, the pregnancy rate drops significantly. The uterus may not be clearly displayed on the ultrasound image due to individual differences. Therefore, it is necessary to reproduce the expertness of physicians on a computer. R. Fanchin et al. made 2-D spatio-temporal images with the vertical and time axis at intervals of 2 frames per second from the obtained ultrasound images of the uterine. 
Table 1 Pregnancy rate by frequency of uterus peristalsis [11].

\begin{tabular}{|c|c|c|c|c|}
\hline \multicolumn{5}{|c|}{ No. of the frequency of uterine peristalsis / $\min$} \\
\hline$\leq 1.0$ & $1.1-2.0$ & $2.1-3.0$ & $3.1-4.0$ & $4.0<$ \\
\hline \hline $58.97 \%$ & $63.55 \%$ & $48.71 \%$ & $6.25 \%$ & $0.00 \%$ \\
\hline
\end{tabular}

The frequency of uterus peristalsis was determined from the time-dependent variation of the interface between the myometrium and the endometrium in the 2-D spatio-temporal image [8]-[10]. L. Zhu et al. measured the frequency of uterus peristalsis from the average of the results of two observers analyzing the ultrasound image of uterus peristalsis for 5 minutes at 4 times speed [11]. D. Meirzon et al. proposed an automated evaluation method by frequency analysis as a computer-aided diagnosis system [12]. However, it is difficult to apply it to the images without strong contrasts between the Region of Interests (ROI) and the uterus.

This paper proposes a method to semi-automatically measure the frequency of uterus peristalsis from ultrasound images. This original version has published [13]. In it, uterine peristalsis was analyzed by Fourier transform for 3 minutes. The frequency of uterine peristalsis was determined as the frequency with the dominant frequency ingredient with maximum value among the frequency spectrums. In this paper, we proposed a method to calculate a fuzzy degree whether uterine peristalsis is the cause of female infertility using fuzzy membership function [14]-[16]. This method makes it possible to quantitatively and objectively evaluate the frequency of uterus peristalsis in the implantation phase for the images without the clear contrast. This method provides the number of frequency of uterus peristalsis, and it enables us to decide the appropriate period of the implantation. This result would reduce the burden on the physician who evaluates the uterine in the implantation phase. This paper is organized as follows. Section 2 describes the materials used here and the proposed method. Section 3 shows the experimental results. Section 4 concludes our technical results.

\section{Materials and Method}

\subsection{Preliminaries}

In general, methods such as inter-frame difference, background difference, and optical flow are often used for moving object detection and grayscale tracking in video. However, it is not easy to detect moving objects by the difference between frames in the ultrasound images used in this study. Figure 1 shows the ultrasound images of the uterus used in this study. Figure 1 shows that the temporal change of the motion is not clear and the change of the brightness value is severe. Moreover, the contour of the ultrasound image is not clear. For these reasons, it was not possible to measure the peristaltic movement frequency from the ultrasound image of the uterus by using the inter-frame difference and optical flow, which are the representative methods of motion detec-

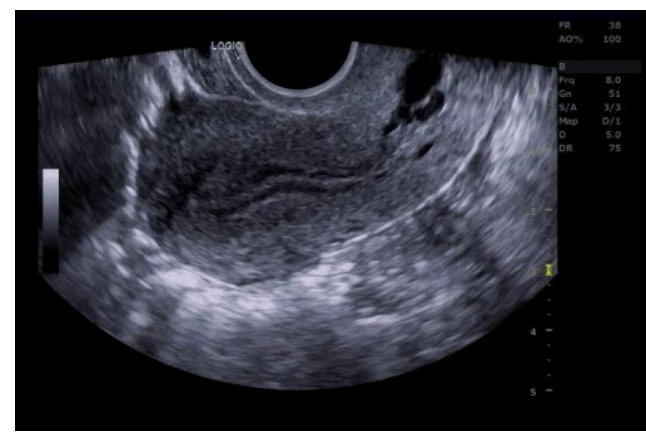

(a) $t=0 \mathrm{sec}$

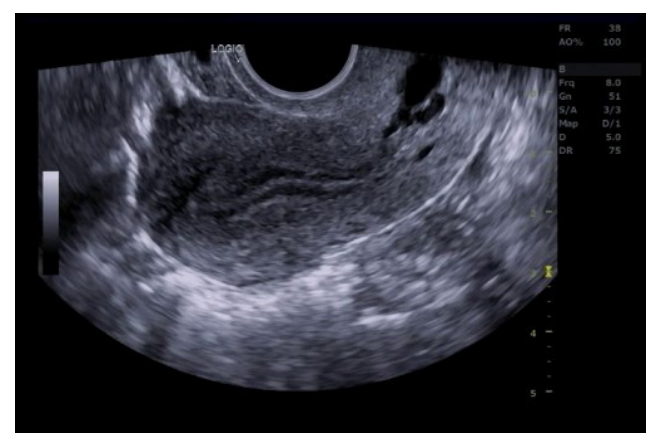

(b) $t=1 \mathrm{sec}$

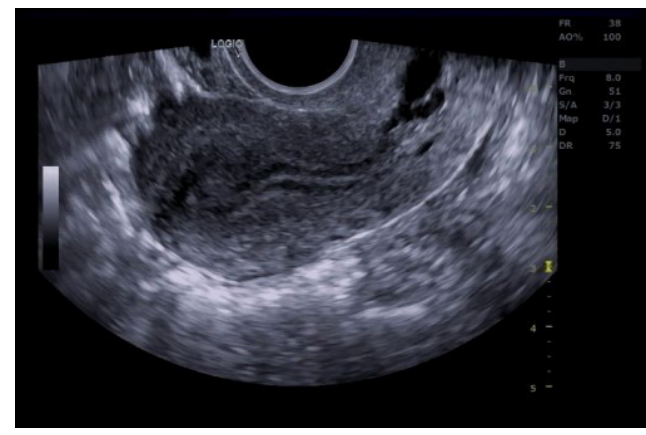

(c) $t=2 \mathrm{sec}$

Fig. 1 Time change of ultrasound image of uterus

tion.

In this study, the ultrasound image of sagittal plane of the uterine in the implantation phase taken in Reproduction Clinic Osaka are employed. The ultrasound image has a $720 \mathrm{px} \times 480 \mathrm{px}$ grayscale MPEG file acquired at a rate of 30 fps by General Electric (GE) Logic 5 system. Figure 2 shows an example of the ultrasound image of the uterine. The area surrounded by the white line in Fig. 2 is the ROI.

\subsection{Method}

Figure 3 shows a flowchart of the analysis method. We explain our proposed method according to Fig. 3 .

\section{Trimming}

Grayscale images are acquired from the movie file, the ROI and its surroundings are trimmed manually as shown in Fig. 4. 


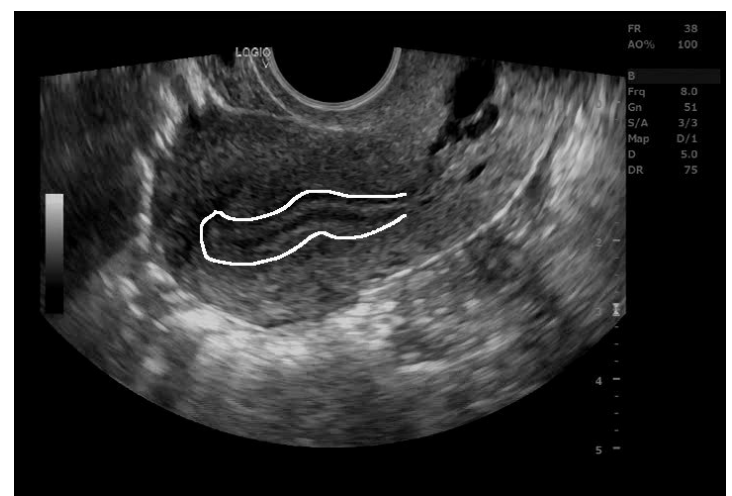

Fig. 2 An actual ultrasound image of the uterine

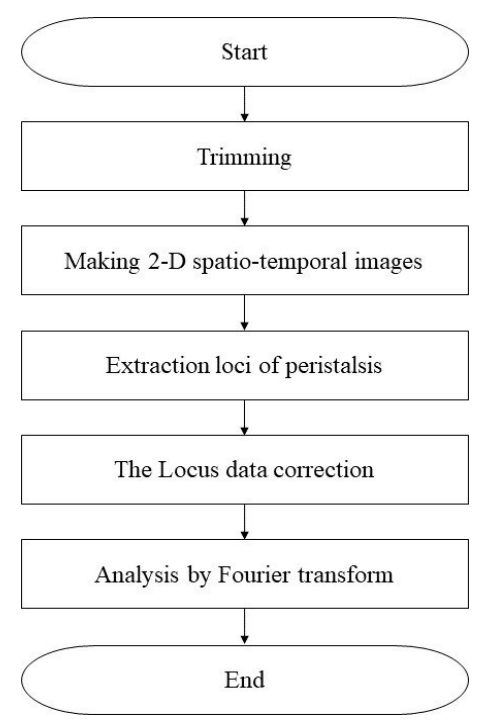

Fig. 3 A flowchart of the anlysis method

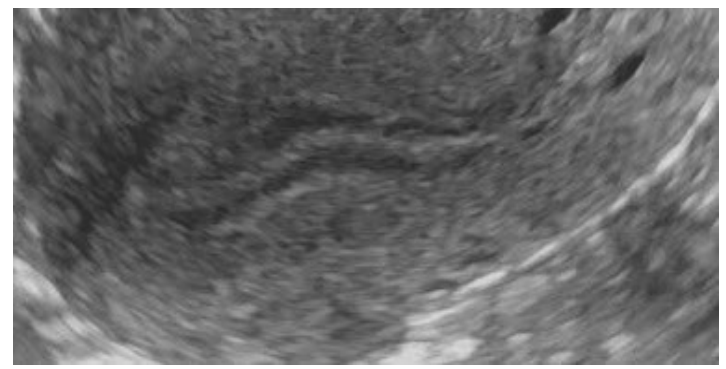

Fig. 4 An example of the trimmed image

\section{Making 2-D spatio-temporal image}

The brightness of the trimmed image is standardized by using the mean and the standard deviation. Five $x$-axis values of the trimmed image are fixed, denoted by $x_{0}^{\prime}, x_{1}^{\prime}, \ldots, x_{4}^{\prime}$ as shown in Fig. 5. An example of the obtained 2-D spatiotemporal image shown in Fig. 6 is made by acquiring the pixels on $x_{i}^{\prime}(i=0,1,2,3$ and 4$)$ at intervals of 10 frames. The value of $x_{0}^{\prime}$ is manually determined, and the values of $x_{1}^{\prime} \sim x_{4}^{\prime}$ were automatically set in the positive $x$-axis direction. The interval between $x_{i}^{\prime}$ and $x_{i}^{\prime}$ was 5 pixel. They

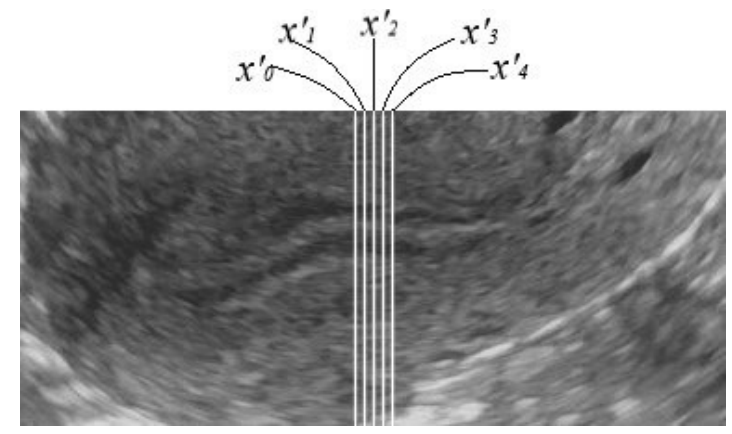

Fig. 5 Points of $x_{i}^{\prime}$ set when creating 2-D spatio-temporal images

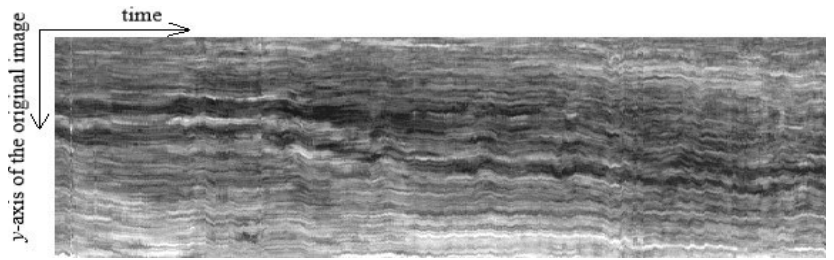

Fig. 6 An example of the 2-D spatio-temporal image

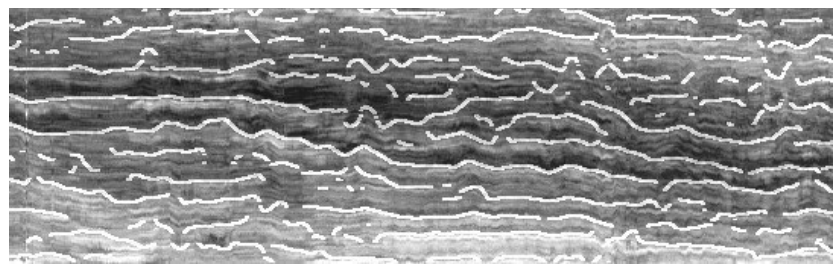

Fig. 7 An example of an automatic extraction result

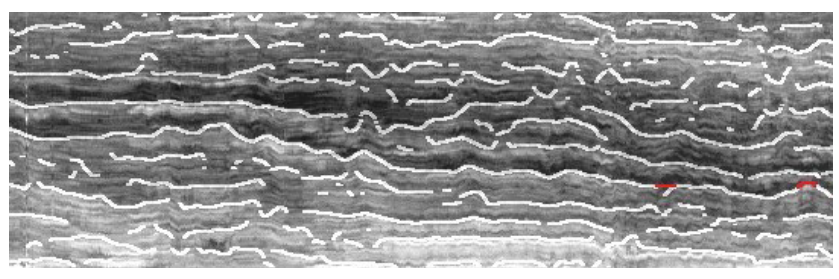

Fig. 8 An example of manual completion (The red lines are the supplemented part)

should clearly be located in uterine peristalsis.

\section{Extraction tracks of uterus peristalsis}

The 2-D spatio-temporal image is filtered in the order of Gaussian filter $(13 \times 13)$, Laplacian filter $(11 \times 11)$, Median filter $(9 \times 9)$. Sobel filter $(3 \times 3)$ is next applied for only $y$-axis edge extraction. The obtained image is binarized by discriminant analysis method. A locus of uterus peristalsis cannot be completely extracted as shown in Fig. 7. Therefore, the lacked part of the locus is manually added as shown in Fig. 8. At this time, at least one locus line must be completed. The left edge of the complemented locus is then determined. The red line in Fig. 9 shows the extracted locus of uterus peristalsis from Fig. 6 . The position of the uterine 


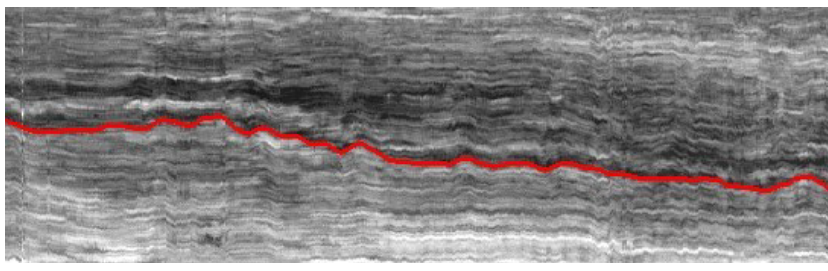

Fig. 9 An example of a locus of uterus peristalsis

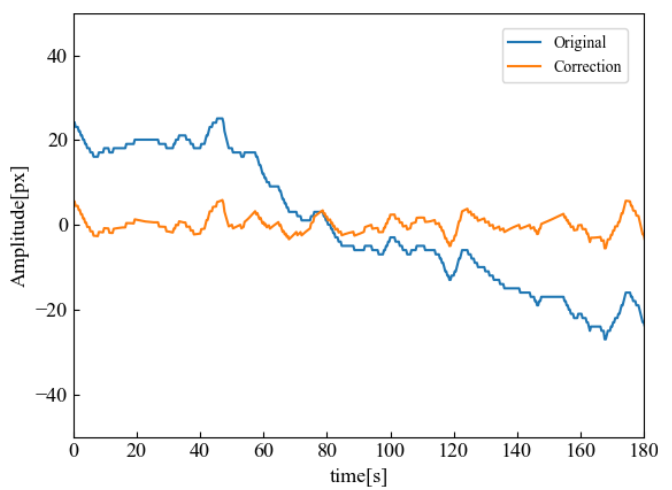

Fig. 10 Comparison between the original locus and the correction locus

is sometimes shifted by movement of the area around the uterus. This fact makes the noise on our frequency domain analysis. Therefore, the shift of the uterine position is adjusted by subtracting the locus line smoothed by the moving average from original locus line. Figure 10 shows the comparison result between the original locus and the obtained locus. This figure shows that the obtained locus is stable and suitable for applying Fourier transform.

\section{Analysis by Fourier transform}

All the trajectory data of uterus peristalsis obtained by the above are Fourier transformed, and the frequency of a given uterus peristalsis is obtained as the common frequency ingredient among the frequency spectrums. We used the Hanning window as the window function. The calculated frequency of uterus peristalsis is compared with the diagnostic result of the physician. It is not easy to watch it with the 1 times speed ultrasound image, since uterus peristalsis has some slow movements. The physician usually examined the ultrasound image at 10 times speed and visually evaluated by his naked eye.

\section{Results}

We evaluated the images for nineteen subjects. On the first subject, Fig. 11 shows the locus of uterus peristalsis corrected by extracting from $2-\mathrm{D}$ spatio-temporal image made from $x_{i}^{\prime}$ in Fig. 5. Figure 12 shows the frequency spectrums of a common frequency ingredient obtained from the average and minimum values of the frequency spectrum of each uterus peristalsis. Furthermore, the moving average for correction was applied at 75 points. We chose the minimum and average values in the frequency spectrum as the com-

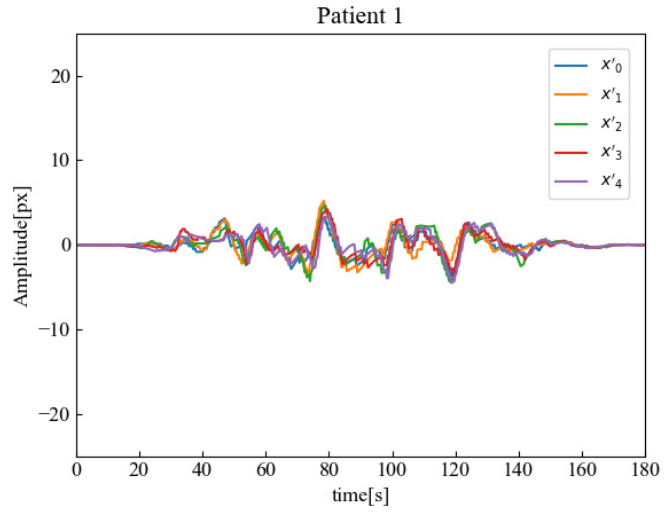

Fig. 11 Loci of uterus peristalsis (Patient 1)

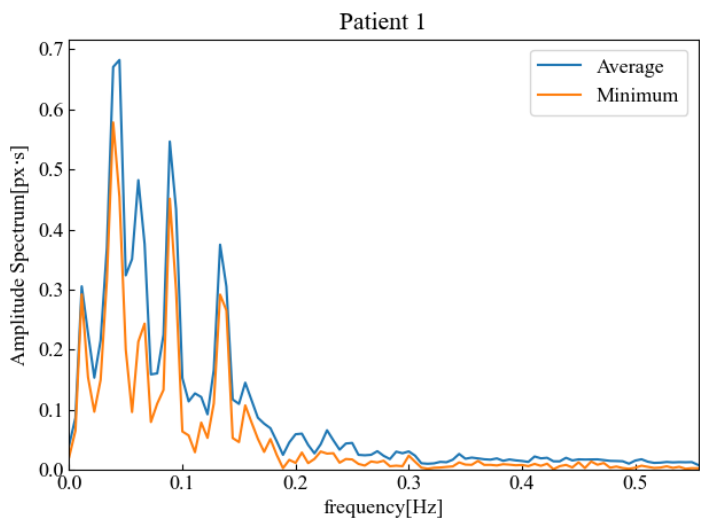

Fig. 12 The frequency spectrum of the common frequency ingredient (Patient 1)

mon frequency ingredient because the low frequency component is primary in our study. From Fig. 12, frequencies with the maximum frequency spectrum were $0.044 \mathrm{~Hz}$ for the spectrum obtained with the average value and $0.039 \mathrm{~Hz}$ for the spectrum obtained with the minimum value. The notations $n_{\text {ave }}$ and $n_{\text {min }}$ denote the number of uterus peristalsis of the average and minimum per minute, respectively. Then, Eqs. (1) and (2) hold.

$$
\begin{aligned}
& n_{\text {ave }} / 60=0.044 \\
& n_{\text {min }} / 60=0.039
\end{aligned}
$$

Then, $n_{\text {ave }}$ was 2.7 , and $n_{\min }$ was 2.3 . This patient's uterine was estimated to have occurred uterus peristalsis 2.7 or 2.3 times per minute. The physician diagnosed this patient's uterus peristalsis as 2.7 times per minute. This diagnostic result equals to the result obtained from the frequency with frequency spectrum obtained with the average value. Table 2 shows the obatained results and physician diagnostic results of all patients. The mean absolutely error (MAE) and the standard error (SE) wer calculated to evaluate the accuracy of the average and the minimum value spectrums. In Table 2, the MAE and the SE of the average and the minimum value spectrums were $1.4 \pm 0.30(\mathrm{MAE} \pm \mathrm{SE})$ and $1.3 \pm 0.29$, respectively.

According to Table 1, the success rate of pregnancy 
becomes extremely low when the peristaltic movement frequency per minute exceeds 3 times. We propose a method to calculated a fuzzy degree whether uterine peristalsis is the cause of the infertility. We define a fuzzy membership function [14]-[16] from Table 1. Figure 13 shows the fuzzy membership function defined by Eq. (3). The function is defined as value about 0.5 when the peristaltic movement frequency per minute is 2.5 and approximatrely 1 when that is over 4 from Table 1 . Thus, the higher degree implies the degree of cause of uterine peristalsis being female infertility.

Table 2 No. of the frequency of uterine peristalsis of all subjects

\begin{tabular}{|c|c|c|c|}
\hline Patient & $\begin{array}{c}\text { True number by } \\
\text { the physician }\end{array}$ & $\begin{array}{c}\text { The number } \\
\text { obtained by } \\
\text { average } \\
\text { spectrum }\end{array}$ & $\begin{array}{c}\text { The number } \\
\text { obtained by } \\
\text { minimum } \\
\text { spectrum }\end{array}$ \\
\hline 1 & 2.7 & 2.7 & 2.3 \\
\hline 2 & 3.3 & 3.3 & 3.3 \\
\hline 3 & 4.0 & 4.7 & 4.7 \\
\hline 4 & 4.0 & 2.6 & 2.6 \\
\hline 5 & 0.67 & 2.7 & 2.0 \\
\hline 6 & 3.3 & 2.6 & 2.6 \\
\hline 7 & 8.0 & 3.6 & 3.3 \\
\hline 8 & 2.0 & 2.0 & 3.0 \\
\hline 9 & 4.0 & 3.0 & 3.3 \\
\hline 10 & 3.0 & 3.0 & 3.0 \\
\hline 11 & 4.0 & 4.0 & 4.0 \\
\hline 12 & 1.3 & 2.3 & 2.3 \\
\hline 13 & 3.3 & 2.7 & 2.7 \\
\hline 14 & 4.7 & 2.7 & 2.7 \\
\hline 15 & 1.0 & 2.3 & 1.3 \\
\hline 16 & 8.0 & 4.3 & 4.6 \\
\hline 17 & 4.0 & 2.0 & 1.7 \\
\hline 18 & 4.0 & 1.7 & 4.6 \\
\hline 19 & 0.67 & 3.6 & 3.6 \\
\hline \multicolumn{2}{|c|}{$\mathrm{MAE} \pm \mathrm{SE}$} & $1.4 \pm 0.30$ & $1.3 \pm 0.29$ \\
\hline
\end{tabular}

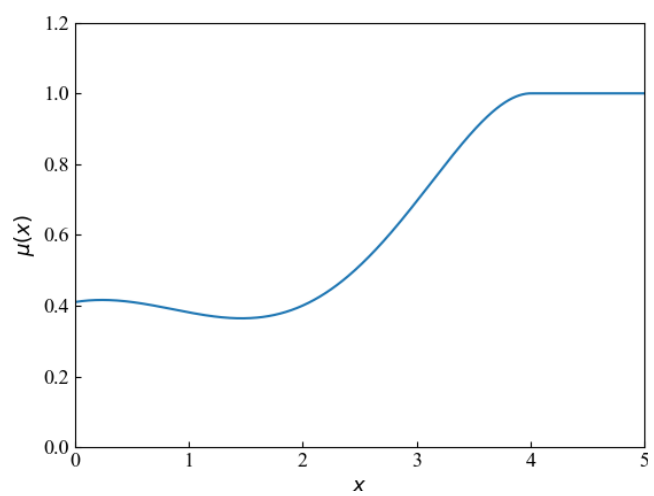

Fig. 13 Fuzzy membership function

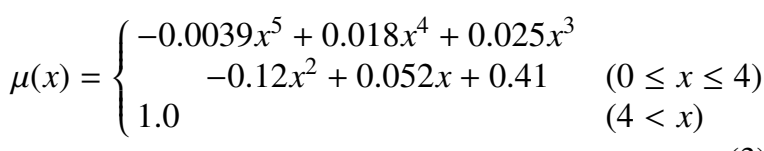

Table 3 shows the fuzzy degrees calculated. This table shows that the degree is smaller if the frequency of uterus peristalsis per minute is less than 3.0.

\section{Conclusions}

In this study, the frequency of uterus peristalsis was analyzed by 2-D spatio-temporal image and Fourier transform. As a result, the frequency of uterine peristalsis was measured as the frequency spectrum of a common frequency component for a given ultrasound image. Here, we quantitatively evaluated the frequency of uterus peristalsis. This study enables us to measure the frequency for the images without enough contrast between the ROI and the others. Here, the traces of uterine peristalsis were done from thinned 2-D spatio-temporal images by manual delineating. Finally, we provide fuzzy degrees for uterine peristalsis. As the results, the fuzzy degrees of Patients $1,5,8,12,15$, and 19 were smaller than the others. We guess that these have problems of the movements. Patient 19 has the big difference between our result with that of physician.

As the future study, it remains that we develop an automatically extraction of the track of uterus peristalsis, and

Table 3 Evaluation result by fuzzy membership function

\begin{tabular}{|c|c|c|c|}
\hline Patient & $\begin{array}{c}\text { Result by the } \\
\text { physician }\end{array}$ & $\begin{array}{c}\text { Result obtained } \\
\text { by average } \\
\text { spectrum }\end{array}$ & $\begin{array}{l}\text { Result obtained } \\
\text { by minimum } \\
\text { spectrum } \\
\end{array}$ \\
\hline 1 & 0.58 & 0.58 & 0.46 \\
\hline 2 & 0.82 & 0.82 & 0.82 \\
\hline 3 & 1.0 & 1.0 & 1.0 \\
\hline 4 & 1.0 & 0.54 & 0.54 \\
\hline 5 & 0.40 & 0.58 & 0.40 \\
\hline 6 & 0.82 & 0.54 & 0.54 \\
\hline 7 & 1.0 & 0.93 & 0.82 \\
\hline 8 & 0.40 & 0.40 & 0.70 \\
\hline 9 & 1.0 & 0.69 & 0.82 \\
\hline 10 & 0.69 & 0.69 & 0.70 \\
\hline 11 & 1.0 & 1.0 & 1.0 \\
\hline 12 & 0.37 & 0.46 & 0.46 \\
\hline 13 & 0.82 & 0.58 & 0.58 \\
\hline 14 & 1.0 & 0.58 & 0.58 \\
\hline 15 & 0.38 & 0.46 & 0.37 \\
\hline 16 & 1.0 & 1.0 & 1.0 \\
\hline 17 & 1.0 & 0.40 & 0.37 \\
\hline 18 & 1.0 & 0.37 & 1.0 \\
\hline 19 & 0.40 & 0.93 & 0.93 \\
\hline
\end{tabular}


a method to reduce the error such as Patient 19.

References

[1] Registration/Investigation Subcommittee, "ART Data Book 2016," Japan Society of Obstetrics and Gynecology, https://plaza.umin.ac. jp/ $/$ jsog-art/2016data_20180930.pdf, accessed March 22. 2021.

[2] OFFICE ON WOMEN'S HEALTH, "A-Z Health Topics "Infertility", Office of the Assistant Secretary for Health, https://www. womenshealth.gov/a-z-topics/infertility, accessed Feb. 13. 2021.

[3] C. Bulletti, D. de Ziegler, V. Polli, L. Diotallevi, E. Del Ferro, and C. Flamigni, "Uterine contractility during the menstrual cycle," Human Reproduction, vol.15, issue suppl 1, pp.81-89, June 2000.

[4] E.A. Lyons, P.J. Taylor, X.H. Zehng, G. Ballard, C.S. Levi, and J.V. Kredentser, "Characterization of subendometrial myometrial contractions throughout the menstrual cycle in normal fertile women," Fertility and Sterility, vol.55, issue 4, pp.771-774, April 1991.

[5] G. Kunz, D. Beil, H. Deininger, L. Wildt, and G. Leyendecker, "The dynamics of rapid sperm transport through the female genital tract: evidence from vaginal sonography of uterine peristalsis and hysterosalpingoscintigraphy," Human Reproduction, vol.11, issue 3, pp.627-632, March 1996.

[6] A. Nakai, K. Togashi, T. Yamaoka, T. Fujiwara, H. Ueda, T. Koyama, H. Kobayashi, T. Kagimura, S. Fujii, and J. Konishi, "Uterine peristalsis shown on cine MR imaging using ultrafast sequence," Journal of Magnetic Resonance Imaging, vol.18, issue 6, pp.726-733, Dec. 2003.

[7] V.A.V. Fornazari, S.A. Vayego, D. Szejnfeld, J. Szejnfeld, and S.M. Goldman, "Functional magnetic resonance imaging for clinical evaluation of uterine contractility," einstein (São Paulo), vol.16, no.1, pp.1-4, April 2018.

[8] R. Fanchin, J.-M. Ayoubi, F. Olivennes, C. Righini, D. de Ziegler, and R. Frydman, "Hormonal influence on the uterine contractility during ovarian stimulation," Human Reproduction, vol.15, issue suppl 1, pp.90-100, June 2000.

[9] R. Fanchin, C. Righini, F. Olivennes, S. Taylar, D. de Ziegler, and R. Frydman, "Uterine contractions at the time of embryo transfer alter pregnancy rates after in-vitro fertilization," Human Reproduction, vol.13, issue 7, pp.1968-1974, July 1998.

[10] R. Fanchin, J.M. Ayoubi, C. Righini, F. Olivennes, L.M. Schönauer, and R. Frydman, "Uterine contractility decreases at the time of blastocyst transfers," Human Reproduction, vol.16, issue 6, pp.1115-1119, June 2001.

[11] L. Zhu, H.S. Che, L. Xiao, and Y.P. Li, "Uterine peristalsis before embryo transfer affects the chance of clinical pregnancy in fresh and frozen-thawed embryo transfer cycles," Human Reproduction, vol.29, issue 6, pp.1238-1243, June 2014.

[12] D. Meirzon, A.J. Jaffa, Z. Gordon, and D. Elad, "A new method for analysis of non-pregnant uterine peristalsis using transvaginal ultrasound," Ultrasound in Obstetrics \& Gynecology, vol.38, issue 2, pp.217-224, Aug. 2011.

[13] R. Nishihara, H. Matsubayashi, T. Ishikawa, and Y. Hata, "Automated Diagnosis of the Frequency of Uterine Peristalsis for Female Infertility," Proc. 2020 IEEE 50th International Symposium on Multiple-Valued Logic (ISMVL), pp.7-11, Miyazaki, Japan, Nov. 2020.

[14] L.A. Zadeh, "Fuzzy Sets," Information and Control, vol.8, issue 3, pp.338-353, June 1965.

[15] D. Dubois and H. Prade, "Fuzzy Sets," in Fuzzy Sets and Systems: Theory and Applications, ed. R. Bellman, pp.9-34, Academic Press, Orlando, 1980.

[16] G.J. Klir and B. Yuan, "FROM ORDINARY (CRISP) SETS TO Fuzzy SETS: A GRAND PARADIGM SHIFT," in FUZZY SETS AND FUZZY LOGIC Theory and Applications, ed. P. Guerrieri, pp.1-33, Prentice Hall, Upper Saddle River, 1995.

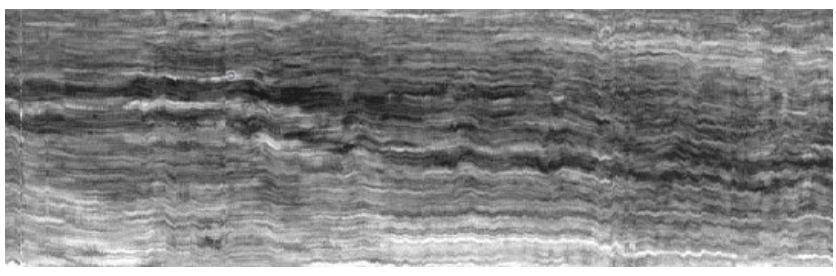

(a) Original image

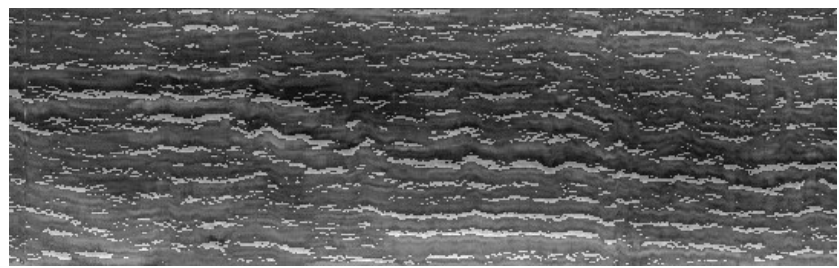

(b) $3 \times 3$

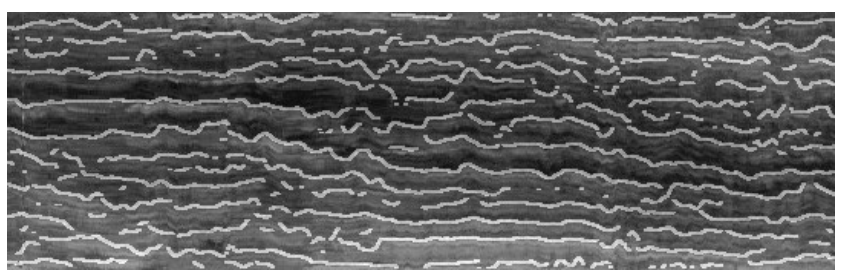

(c) $11 \times 11$

Fig. A- 1 Effect of Laplacian filter + Sobel filter (only y-axis)

\section{Appendix}

Our process of applying the Sobel filter after applying the Laplacian filter in our method is shown as appendix. The Laplacian filter takes the second derivative of the image, and the Sobel filter takes the first derivative of the image. In other words, performing the filtering process in the order of the Laplacian filter and the Sobel filter will lead the same as taking the third derivative of the image. Then, Eq. (4) holds.

$$
\frac{\partial}{\partial y}\left(\frac{\partial^{2}}{\partial x^{2}}+\frac{\partial^{2}}{\partial y^{2}}\right)
$$

Here, the Laplacian filter used here has $11 \times 11$ size, and the Sobel filter has $3 \times 3$. They have a big difference in their sizes. Then, the Sobel filter performs local processing on the Laplacian filter, and therefore Eq. (4) does not hold. Figure A-1 (b) and (c) shows the result of applying the Laplacian filter and then the Sobel filter (only $y$-axis edge extraction) with $3 \times 3$ and $11 \times 11$ sizes of the Laplacian filter, respectively. Figure A. 1 (c) demonstrates that the edge image is clearly extracted for the original image. 


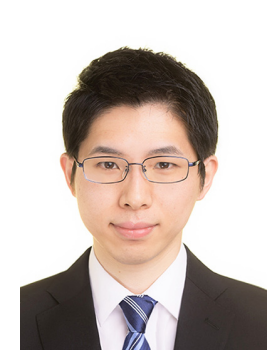

Ryosuke Nishihara received the B.E. degrees in from University of Hyogo in 2020. He now with Graduate School of Simulation Studies. His research interest is in medical diagnosis system.

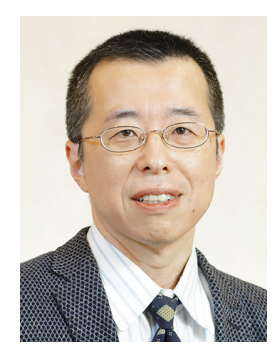

Hidehiko Matsubayashi

completed his M.D. at the Keio University, Tokyo, Japan, in 1988, and obtained his Ph.D. at the Keio University in 1998. He worked for reproductive immunology laboratory in Methodist Hospital, Indianapolis, IN, USA, as a research fellow from 1996 to 1998. He was associate professor at the Tokai University, Kanagawa, Japan, from 2005 to 2012. At present he is a supervisor of Reproduction Clinic, Japan, since 2013.

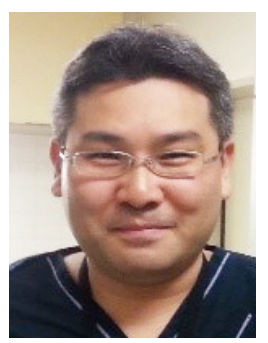

Tomomoto Ishikawa received an M.D. in 2000 and a Ph.D. in 2006, both from Kobe University, Japan. He started his fellowship at Kobe University Hospital and transferred to the Population Council, Rockefeller University, USA. He completed his fellowship in the area of male infertility and reproductive function. His expertise in urology and male reproductive medicine has been recognized by national and international authorities with invitations from the American Urologic Association, American Society of Andrology, and Japanese urologic and reproductive societies to speak on issues of male infertility treatment. He is now CEO and Urologist-in-Chief at the Reproduction Clinic, Osaka and Tokyo and Ishikawa Hospital. He is an internationally acclaimed expert in the treatment of male infertility, especially the interface of male factor treatment with assisted reproductive techniques (ART).

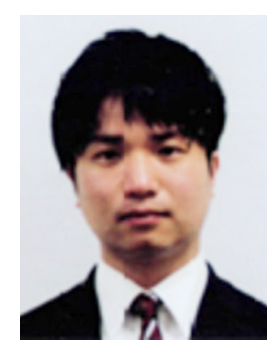

Kentaro Mori received the B.E. in National Institute of Technology, Maizuru College in 2016, the M. Simulation studies degrees in Graduate School of Simulation studies, University of Hyogo in 2018. Currently, he is an assistant professor at Dept. of Electrical and Computer Engineering, National Institute of Technology, Maizuru College.

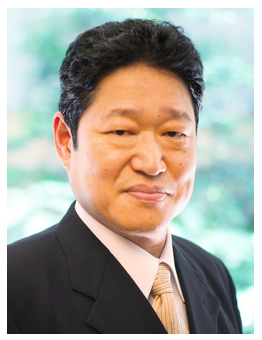

Yutaka Hata received the B.E. degree in 1984, the M.E. degree in 1986 and the Ph.D. in 1989 all from Himeji Institute of Technology, Japan. He is currently a Professor in the Graduate School of Simulation Studies, University of Hyogo, Japan. He spent one year in BISC Group, University of California at Berkeley from 1995 to 1996 as a visiting scholar. His research interests are in medical system, health monitoring system, fuzzy system and Immune system. He received about 15 international awards such as the Franklin V. Taylor Best Paper Award (IEEE SMC 2009), etc. He is 5 Journal editors including IEEE Trans. SMC-Systems. $\mathrm{He}$ is an IEEE Fellow. 